\title{
Olmesartan reduces oxidative stress in the brain of stroke-prone spontaneously hypertensive rats assessed by an in vivo ESR method
}

\begin{abstract}
Shuichiro Araki ${ }^{1}$, Yoshitaka Hirooka ${ }^{1}$, Takuya Kishi ${ }^{1}$, Keiji Yasukawa ${ }^{2}$, Hideo Utsumi ${ }^{2}$ and Kenji Sunagawa ${ }^{1}$
We previously showed that oxidative stress in the brain is involved in the neural mechanisms of hypertension. Therefore, olmesartan, an angiotensin type 1 receptor blocker, might affect oxidative stress in the brains of stroke-prone spontaneously hypertensive rats (SHRSP). Here, we evaluated the effects of olmesartan treatment using an in vivo electron spin resonance (ESR)/spin probe technique. Two groups of SHRSP were treated with either olmesartan $\left(10 \mathrm{mg} \mathrm{kg}^{-1} \mathrm{day}^{-1}\right)$ or hydralazine (Hyd, $20 \mathrm{mg} \mathrm{kg}^{-1}$ day $^{-1}$ )/hydrochlorothiazide (HCT, $4.5 \mathrm{mg}^{-1} \mathrm{~kg}$ day $^{-1}$ ) for 30 days ( $n=5$ for each). Systolic blood pressure decreased similarly in both groups after treatment. Heart rate and urinary norepinephrine (NE) excretion increased in rats treated with $\mathrm{Hyd} / \mathrm{HCT}$, but not in those treated with olmesartan. The in vivo ESR signal decay rates of the blood-brain barrierpermeable spin probe methoxycarbonyl-PROXYL were significantly higher in SHRSP brains than in age-matched normotensive Wistar-Kyoto rat brains $(P<0.01 ; n=6$ for each). Olmesartan attenuated the ESR signal decay rates in SHRSP brains, but $\mathrm{Hyd} / \mathrm{HCT}$ did not. Intracerebroventricular infusion of active form of olmesartan, RNH-6270, reduced blood pressure and NE excretion, and the ESR signal decay rate was reduced in SHRSP brains. These findings indicate that olmesartan has anti-oxidative property in the brain without stimulating reflex-mediated sympathetic activity in SHRSP.
\end{abstract}

Hypertension Research (2009) 32, 1091-1096; doi:10.1038/hr.2009.160; published online 18 September 2009

Keywords: angiotensin receptor blocker; brain; oxidative stress; sympathetic nervous system

\section{INTRODUCTION}

Angiotensin type 1 (AT1) receptors within the brain are important for central autonomic regulation of blood pressure. ${ }^{1-3}$ AT1 receptors are rich in the specific brain nuclei that regulate sympathetic nervous activity, ${ }^{1-4}$ such as the anteroventral third ventricle, paraventricular nucleus of the hypothalamus, nucleus tractus solitarius and the rostral ventrolateral medulla (RVLM), ${ }^{2-4}$ where the vasomotor center is located, ${ }^{3}$ of the brainstem, thereby controlling blood pressure. ${ }^{3,4}$ Recent studies suggest that the systemic administration of AT1receptor blockers also act on AT1 receptors within the brain, ${ }^{5}$ thereby modifying blood pressure control. ${ }^{6-10}$

Oxidative stress in the brain increases blood pressure through activation of the sympathetic nervous system. ${ }^{11-14}$ The major source of reactive oxygen species (ROS) is nicotinamide adenine dinucleotide phosphate $(\mathrm{NAD}(\mathrm{P}) \mathrm{H})$ oxidase, $^{12,15,16}$ which is activated by AT1 receptor stimulation. AT1 receptor expression levels in the brain, such as in the RVLM, are upregulated in hypertensive animal models compared with normotensive controls. ${ }^{17,18}$ Olmesartan is a strong AT1-receptor blocker with a high degree of insurmountability. ${ }^{19,20}$ We hypothesized that systemic administration of olmesartan reduces oxidative stress in the brain, as well as in the peripheral vasculature, ${ }^{21}$ and that this antioxidant action may account for the absence of reflex-induced sympathoexcitation after treatment with olmesartan. To examine this, we applied the technique of in vivo electron spin resonance (ESR) spectroscopy, ${ }^{22}$ because the ESR method is a powerful technique for evaluating oxidative stress noninvasively ${ }^{22,23}$ and is useful for assessing drug effects when applied in vivo. ${ }^{24}$ Using this technique, we examined the effects of chronic oral administration of olmesartan on oxidative stress in the brain of stroke-prone spontaneously hypertensive rats (SHRSP), as well as on blood pressure, heart rate $(\mathrm{HR})$ and urinary norepinephrine (NE) excretion. To examine the direct action of olmesartan on the brain, we also evaluated the effect of intracerebroventricullar administration of RNH-6270 (an active form of olmesartan) on these variables.

\section{METHODS}

Animals and general procedures

All procedures and animal care were approved by the Committee on Ethics of Animal Experiments, Kyushu University Graduate School of Medical and Pharmaceutical Sciences, and performed in accordance with the Guidelines for Animal Experiments at Kyushu University. Male SHRSP and Wistar-Kyoto (WKY) rats (11 weeks old, Hamamatsu, Japan) were fed standard chow and

${ }^{1}$ Department of Cardiovascular Medicine, Kyushu University Graduate School of Medical Sciences, Kyushu University, Maidashi, Higashi-ku, Fukuoka, Japan and ${ }^{2}$ Department of Bio-Functional Science, Graduate School of Pharmaceutical Sciences, Kyushu University, Maidashi, Higashi-ku, Fukuoka, Japan

Correspondence: Dr Y Hirooka, Department of Cardiovascular Medicine, Kyushu University Graduate School of Medical Sciences, 3-1-1 Maidashi, Higashi-ku, Fukuoka 8128582, Japan.

E-mail: hyoshi@cardiol.med.kyushu-u.ac.jp

Received 24 June 2009; revised and accepted 6 August 2009; published online 18 September 2009 
had free access to drinking water. Treatment was started when the rats were 12 weeks old and continued for 30 days. The rats received either olmesartan (olmesartan medoxomil, CS-866, Daiichi-Sankyo, Tokyo, Japan; $10 \mathrm{mg} \mathrm{kg}^{-1} \mathrm{day}^{-1}$, in $0.5 \%$ sodium carboxymethylcellulose colloidal solution) once a day by oral gavage, a solution of hydralazine (Sigma Chemical, St Louis, MO, USA; Hyd, $20 \mathrm{mg} \mathrm{kg}^{-1} \mathrm{day}^{-1}$ ) and hydrochlorothiazide (Sigma; HCT, $4.5 \mathrm{mg} \mathrm{kg}^{-1} \mathrm{day}^{-1}$ ) in their drinking water (Hyd/HCT), or no drugs (no treatment).

\section{Measurements of blood pressure, HR and urinary NE excretion} Systolic blood pressure (SBP) and HR were measured by tail-cuff plethysmography (BP-98A, Softron, Tokyo, Japan) every other day during the study period. Urine was collected individually for $24 \mathrm{~h}$ in a metabolic cage on the days before and at the end of each treatment. Urinary NE concentration was measured by high-performance liquid chromatography to calculate urinary NE excretion as a marker of sympathetic nervous system activity. ${ }^{13,14,16}$

\section{NADPH oxidase activity}

Nicotinamide adenine dinucleotide phosphate (NADPH) oxidase activity was evaluated using lucigenin chemiluminescence as described elsewhere, ${ }^{25}$ in membrane fraction proteins. Briefly, a deeply anesthetized rat (sodium pentobarbital, $75 \mathrm{mg} \mathrm{kg}^{-1}$, intraperitoneal) was perfused transcardially with $200 \mathrm{ml}$ chilled saline. The brain was quickly removed and divided into four parts: the cerebral cortex, cerebellum, hypothalamus and the medulla oblongata. All tissues were homogenized in ice-chilled $50 \mathrm{~mm}$ phosphate buffer $(\mathrm{pH} 7.4$ ) containing proteinase inhibitors (Complete Mini, Roche, Basel, Switzerland) and $1 \mathrm{~mm}$ phenylmethylsulfonyl fluoride. The tissue homogenates were centrifuged at $24000 \mathrm{~g}$ for $20 \mathrm{~min}$ at $4{ }^{\circ} \mathrm{C}$, and then the supernatants were subjected to $100000 \mathrm{~g}$ spin for $60 \mathrm{~min}$ at $4{ }^{\circ} \mathrm{C}$ to collect plasma and microsome membranes. The pellets were resuspended and protein concentrations were measured with a BCA assay kit (Pierce Chemical, Rockford, IL, USA). Luminescence measurements were performed as previously described. ${ }^{16}$ Data were expressed as relative light units per minute per microgram protein.

\section{In vivo ESR spectroscopy}

We used methoxycarbonyl (MC)-PROXYL, a nitroxyl radical species, as the blood-brain barrier-permeable spin probe because of its lipophilicity. ${ }^{26-28} \mathrm{MC}$ PROXYL undergoes a biological reaction in vivo to non-radical derivatives such as hydroxylamine. ${ }^{29}$ In addition, increased ROS precipitate the reaction nonenzymatically. ${ }^{30}$ This provides the basic mechanism for the in vivo ESR method; that is, increased ROS production increases the in vivo ESR signal decay rate in comparison with an appropriate control value.

An anesthetized rat (sodium pentobarbital, $50 \mathrm{mg} \mathrm{kg}^{-1}$, intraperitoneal) was placed on a hand-made rat carrier. MC-PROXYL solution $\left(150 \mathrm{mmoll}^{-1}\right.$ in saline, $0.33 \mathrm{mmol}$ per kg body weight) was injected into the tail vein of the rat, and then the carrier was slid into the resonator cavity so that the head of the rat, the area between interorbital and interaural lines, was located in the ESRdetecting area (coaxial discoid space; $6 \mathrm{~cm}$ in diameter, $1.5 \mathrm{~cm}$ in depth). Measurement was immediately started using $300-\mathrm{MHz}$ ESR spectroscopy (JEOL, Tokyo, Japan) under the following conditions: radiowave frequency, $300 \mathrm{MHz}$; radiowave power, $2.5 \mathrm{~mW}$; field modulation width, $0.1 \mathrm{mT}$; field modulation frequency, $100 \mathrm{kHz}$; scan rate, $0.083 \mathrm{mTs}^{-1}$; scan width, $1.0 \mathrm{mT}$; time constant, $0.1 \mathrm{~s}$; and accumulation number, 5 .

Signal intensity, defined as the height of the center line of a spectrum (Figure 1a), was obtained using an averaged spectrum that was processed with consecutively accumulated 5 spectra within a 60 -s interval. The measurements were collected for $5 \mathrm{~min}$. The natural logarithmic values of the data were plotted as a function of time, and the signal decay rate was determined by the negative slope of the linear regression of the plots, which had good linearity (Figure 1b).

\section{Effect of antioxidants on in vivo ESR signal decay rate}

Experiments of the in vivo ESR spectroscopy were conducted with pharmacological interventions; pretreatment with apocynin ${ }^{31}$ (Sigma, $5 \mathrm{mg} \mathrm{kg}^{-1}$, intravenous) 20 mins before in vivo ESR spectroscopy, the use of MC-PROXYL $(0.33 \mathrm{mmol}$ per $\mathrm{kg}$ body weight $)$ spin probe solution that contained a

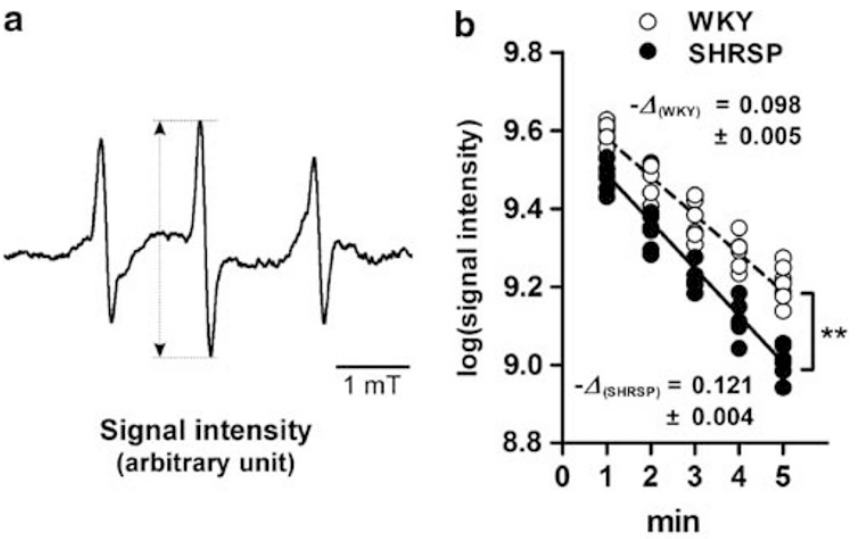

C

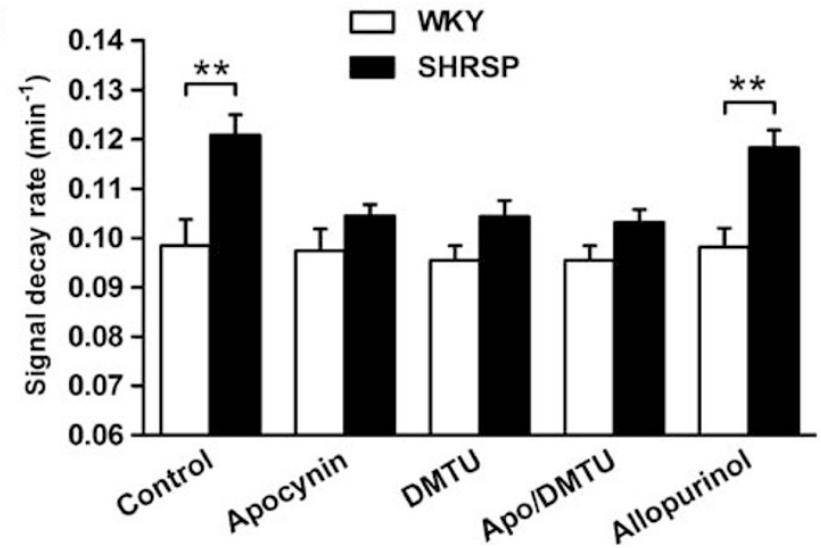

Figure 1 The in vivo electron spin resonance (ESR) method. (a) The ESR spectrum in the head of an anesthetized rat after intravenous injection of methoxycarbonyl (MC)-PROXYL into the tail vein showed a characteristic triplet set of peaks; the pattern was unchanged during measurement, whereas signal intensity, defined as the top-to-bottom height of the center peak of the triplet, decreased with time. (b) The natural logarithmic values of 60-s averages of signal intensity were consecutively plotted as a function of time for $5 \mathrm{~min}$; the signal decay rate was defined as the negative slope $(-4)$ of the linear regression of these plots, which had good linearity. The signal decay rate in stroke-prone spontaneously hypertensive rats (SHRSP) was increased compared with that in age-matched Wistar-Kyoto (WKY) rats; the difference was small, but statistically significant. ${ }^{*} P<0.01, n=6$ for each. (c) The effect of antioxidants on in vivo ESR signal decay rate. Increased signal decay rate in SHRSP (control) was decreased by pretreatment with apocynin (apocynin), or by coadministration of MCPROXYL with dimethylthiourea (DMTU), to comparable levels of those of WKY. Pharmacological intervention in combination with apocynin and DMTU (Apo/DMTU) resulted in no further decrease from respective single treatment. Pretreatment with allopurinol did not affect the increased in vivo ESR signal decay rate in SHRSP (allopurinol). ${ }^{* *} P<0.01$.

dimethylthiourea ${ }^{32}$ (Sigma, $1.0 \mathrm{mmol}$ per kg body weight), or combination of these two interventions. Pretreatment with allopurinol ${ }^{33}$ (Sigma, $100 \mathrm{mg} \mathrm{kg}^{-1}$, intraperitoneal) was also performed $30 \mathrm{~min}$ before in vivo ESR spectroscopy.

\section{Chronic intracerebroventricular infusion of RNH-6270}

Under sodium pentobarbital anesthesia $\left(50 \mathrm{mg} \mathrm{kg}^{-1}\right.$, intraperitoneal), the rat's head was fixed in a stereotaxic frame. A 28-gauge stainless steel cannula (Alzet, DURECT Corp., Cupertino, CA, USA) was chronically implanted in the left lateral cerebral ventricle (coordinates with respect to bregma: ${ }^{34} 0.6 \mathrm{~mm}$ posterior, $1.5 \mathrm{~mm}$ lateral and $4.0 \mathrm{~mm}$ ventral from the skull surface) and fixed to the skull with acrylic cement. The placement was verified by post-mortem injection 
of methylene blue through the L-shaped cannula. Infusion of RNH-6270 at $100 \mu \mathrm{g} \mathrm{day}^{-1}\left(390-410 \mu \mathrm{g} \mathrm{kg}^{-1} \mathrm{day}^{-1}\right.$ ) or vehicle (artificial cerebrospinal fluid containing $\left.0.8 \% \mathrm{NaHCO}_{3}\right)$ was initiated with osmotic minipump $\left(12 \mu \mathrm{lday}^{-1}\right.$, model 2002, Alzet), which were implanted subcutaneously and connected to the intracerebroventricular (ICV) cannula.

\section{Statistical analysis}

All values are expressed as mean \pm s.e. A two-way analysis of variance with Bonferroni post hoc tests was used to compare the SBP and HR, urinary NE excretion, ESR signal decay rate, NADPH oxidase activity and western blot densitometry between groups. A paired $t$-test was performed to compare the urinary NE excretion and ESR signal decay rate between before and after treatment. Comparisons between any two mean values were carried out using a two-tailed unpaired $t$-test with Welch's correction. Differences were considered to be statistically significant when $P$ value was $<0.05$.

\section{RESULTS}

Effect of treatments on SBP, HR and urinary NE levels

Olmesartan significantly decreased SBP to as low as that induced by Hyd/HCT (Figure 2). HR was sporadically higher during treatment in the Hyd/HCT group compared with the olmesartan and untreated groups (Figure 2). Urinary NE excretion was significantly increased only in the Hyd/HCT group (Figure 3a), consistent with the HR increase during treatment.

Effect of olmesartan on oxidative stress in brain as measured by the in vivo ESR method

We successfully obtained the in vivo ESR signal decay rate in the head of rats using a blood-brain barrier-permeable spin probe (Figure 1a). The signal decay rate in the brain of SHRSP was increased compared with age-matched normotensive WKY controls $(0.121 \pm 0.004$ vs.

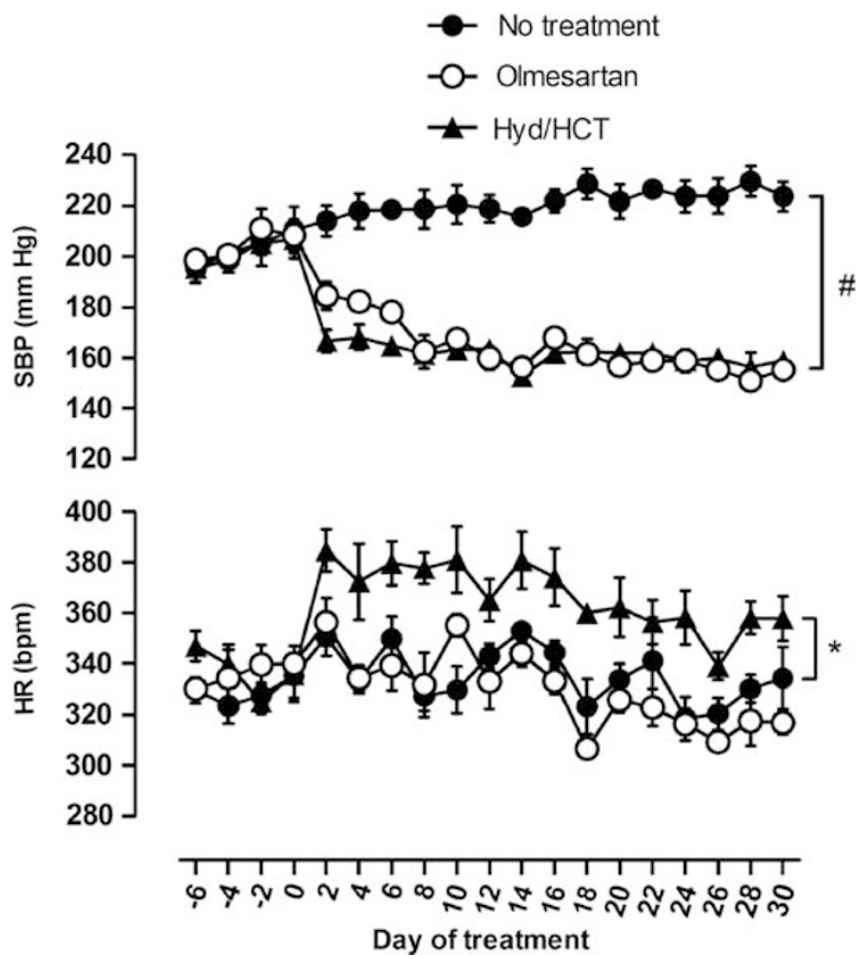

Figure 2 The effect of olmesartan on systolic blood pressure (SBP) and heart rate (HR). Time course of SBP and HR in the groups during the study period. ${ }^{\#} P<0.001$ vs. SBP of the no-treatment group for both the olmesartan and hydralazine (Hyd)/hydrochlorothiazide (HCT) groups; ${ }^{*} P<0.05$ vs. HR of the no-treatment group for the Hyd/HCT group.
$0.098 \pm 0.005$ arbitrary unit (a.u.), $P<0.01$; Figure 1 b). The increased signal decay rate in SHRSP (Figure 1c) was attenuated to a comparable level to that of WKY by pretreatment with apocynin $(0.104 \pm 0.002$ vs. $0.097 \pm 0.004$, a.u., SHRSP vs. WKY, not significant), or coadministration of the spin probe with a potent hydroxyl radical scavenger dimethylthiourea $(0.104 \pm 0.003$ vs. $0.095 \pm 0.003$, a.u., SHRSP vs. WKY, not significant). Pharmacological interventions in combination with pretreatment with apocynin and coadministration of dimethylthiourea with the spin probe did not result in further decrease in the in vivo ESR signal decay rate $(0.103 \pm 0.003$ vs. $0.095 \pm 0.003$, a.u., SHRSP vs. WKY, not significant). Pretreatment with allopurinol, xanthine oxidase inhibitor, did not attenuated the increased signal decay rate in SHRSP brain $(0.118 \pm 0.004$ vs. $0.098 \pm 0.004$, a.u., SHRSP vs. WKY, $P<0.01)$. Figure $3 \mathrm{~b}$ shows the changes in the in vivo ESR signal decay rate after the treatments in SHRSP, indicating a significant decrease by the treatment with olmesartan $(0.102 \pm 0.004$ vs. $0.121 \pm 0.003$, a.u., after $v$ s. before treatment, $P<0.05)$, but not
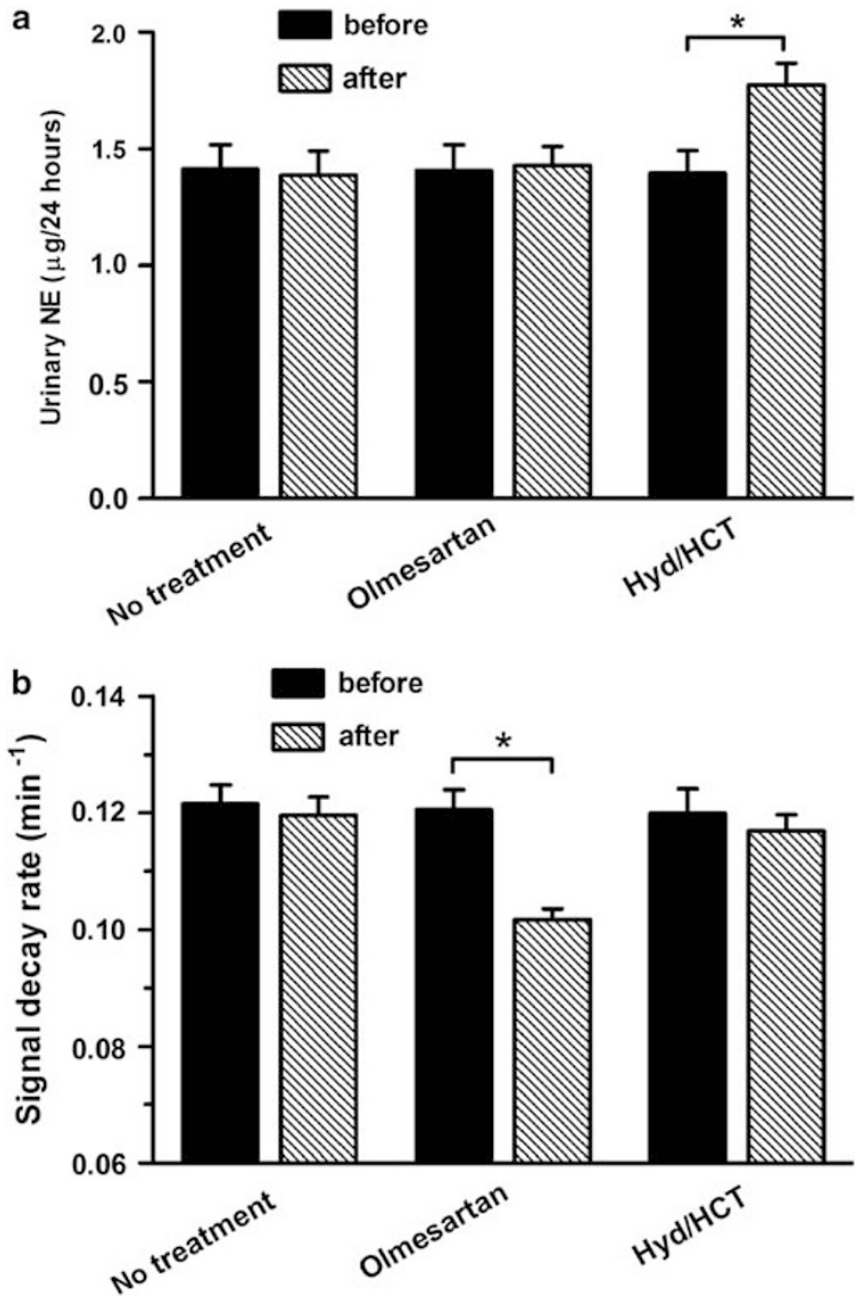

Figure 3 (a) The effect of olmesartan on urinary norepinephrine (NE) excretion. Urinary NE excretion over $24 \mathrm{~h}$, used as a marker of sympathetic activity, was increased only in the hydralazine (Hyd)/hydrochlorothiazide (HCT) group. ${ }^{*} P<0.05$. (b) The effect of olmesartan on the in vivo electron spin resonance (ESR) signal decay rates. In vivo ESR signal decay rates were measured on the days before and after treatment in each rat. The signal decay rate was significantly decreased after treatment with olmesartan, whereas it was unchanged after treatment with $\mathrm{Hyd} / \mathrm{HCT}$ or no-treatment. ${ }^{*} P<0.05$. 
with Hyd/HCT $(0.117 \pm 0.003$ vs. $0.120 \pm 0.004$, a.u., after vs. before treatment, not significant).

\section{Effect of olmesartan on NADPH oxidase activity in the brain}

We then examined whether olmesartan decreased NADPH oxidase activity in the brains of SHRSP, which was thought to be a major source of ROS for oxidative stress (Figure 4). NADPH-dependent superoxide production in the membrane fractions of the cerebral cortex, hypothalamus and medulla oblongata were significantly increased in SHRSP compared with those in WKY (cerebral cortex, $7.04 \pm 1.12$ vs. $2.09 \pm 0.37$; cerebellum, $1.29 \pm 0.25$ vs. $0.46 \pm 0.10$; hypothalamus, $5.67 \pm 1.11$ vs. $1.99 \pm 0.48$; medulla, $9.68 \pm 1.23$ vs. $6.31 \pm 0.45$; RLU $\mathrm{min}^{-1}$ per mg protein, untreated SHRSP vs. WKY, $n=5, P<0.05$ except the cerebellum). Olmesartan treatment significantly attenuated the increase to a level as low as that in WKY (cerebral cortex, $3.00 \pm 0.73$; cerebellum, $0.56 \pm 0.16$; hypothalamus, $1.84 \pm 0.33$; medulla, $5.26 \pm 0.85$; RLU $\min ^{-1}$ per mg protein, $n=5$ ). Although there was a similar trend of increased superoxide production in the cerebellum of untreated SHRSP, the increase was not significant, which may have been due to the low levels of superoxide in the cerebellum such that the relatively large amount of variance prevented the effect from reaching statistical significance.

\section{Central effect of RNH-6270 on cardiovascular responses and} oxidative stress in the brain

To test whether olmesartan acts on the brain to reduce sympathetic activity and blood pressure in SHRSP, chronic ICV infusion of the active form of olmesartan, RNH-6270, was carried out. ICV RNH-6270 significantly reduced SBP and $\mathrm{HR}$, and urinary NE excretion levels (Figure 5) compared with those values in ICVvehicle-treated SHRSP. The in vivo ESR signal decay rate was

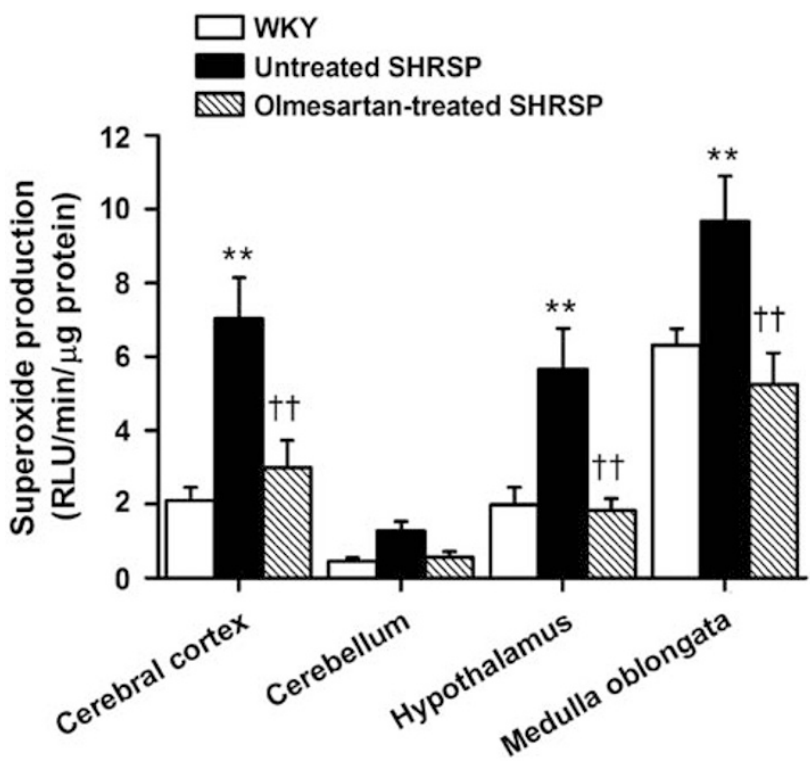

Figure 4 The effect of olmesartan on nicotinamide adenine dinucleotide phosphate (NADPH) oxidase activity in the brain. NADPH oxidase activity was estimated using lucigenin-enhanced chemiluminescence in membrane fractions from the cerebral cortex, cerebellum, hypothalamus and medulla oblongata. NADPH-dependent superoxide production was significantly increased in the brain tissues of stroke-prone spontaneously hypertensive rats (SHRSP) compared with Wistar-Kyoto (WKY) rats in all regions examined, except for the cerebellum, in which superoxide production levels were very low in this assay. The increases were significantly attenuated by treatment with olmesartan. ${ }^{* *} P<0.01$ vs. WKY; ${ }^{\dagger} P<0.01$ vs. untreated SHRSP. significantly decreased after chronic treatments with ICV-RNH-6270, but not ICV-vehicle (Figure 5).

\section{DISCUSSION}

In this study, oral treatment with olmesartan, an AT1-receptor blocker, in SHRSP for 1 month reduced the increased oxidative stress in the brain assessed by an in vivo ESR method using the blood-brain barrier-permeable nitroxyl compound, MC-PROXYL, as the spin probe. Unlike treatment with Hyd and HCT, oral treatment with olmesartan did not elicit a baroreflex-mediated increase in HR and sympathetic nervous system activity. ICV treatment with an active form of olmesartan reduced oxidative stress in the brain in association with decreases in SBP, HR and urinary NE excretion. These results suggest that oral treatment with olmesartan has an anti-oxidative effect on the brain in SHRSP.

The in vivo ESR spectroscopy technique we used to assess oxidative stress in the brain has advantages over other methods of assessing oxidative stress, such as measuring thiobarbituric acid-reactive substances, 8-isoprostane, and so on, because ESR spectroscopy is more direct. ${ }^{35}$ Stable nitroxyl radicals such as PROXYLs are widely used as spin probes to evaluate the generation of ROS in vivo. ${ }^{36}$ The signal decay rates of the nitroxyl radical are enhanced by ROS during oxidative stress. ${ }^{30} \mathrm{MC}$-PROXYL has good blood-brain barrier permeability and more specific distribution in the brain compared with other nitroxyl radicals. ${ }^{27,28,36}$ Thus, the use of MC-PROXYL as the spin probe allowed us to assess oxidative stress in the brain in vivo. A previous study using a similar technique showed that oxidative stress in the brain is enhanced in SHR or SHRSP. ${ }^{37}$ We confirmed the BBB permeability of MC-PROXYL with ESR in the brains of WKY and SHRSP (data not shown). In this study, we show that the in vivo ESR method is useful for noninvasive evaluation of the effect of antihypertensive drugs on the brain oxidative stress in a hypertensive rat model.

Unlike Hyd and HCT, olmesartan did not elicit a baroreflex-mediated increase in HR and sympathetic nervous system activity, despite similar reductions in blood pressure. We selected the dose of $10 \mathrm{mg} \mathrm{kg}^{-1} \mathrm{day}^{-1}$ of olmesartan because this dose is commonly used to reduce blood pressure in hypertensive animal models. ${ }^{38}$ Consistent with our observations, oral administration of olmesartan $\left(10 \mathrm{mg} \mathrm{kg}^{-1} \mathrm{day}^{-1}\right)$ also does not increase HR in SHR. ${ }^{38}$ We did not explore the precise mechanisms by which olmesartan prevents baroreflex-mediated tachycardia and sympathoexcitation. It is possible, however, that olmesartan affects the brain, thereby inhibiting the sympathetic nervous system, at least in part, by reducing oxidative stress in the autonomic nuclei, such as the paraventricular nucleus of the hypothalamus, nucleus tractus solitarus ${ }^{39}$ and RVLM. ${ }^{3,5,13,16}$ We previously showed that increased oxidative stress in the RVLM is involved in the neural mechanisms of hypertension in SHRSP. ${ }^{13}$ Angiotensin II (Ang II) binds AT1 receptors, then activates the $\mathrm{NAD}(\mathrm{P}) \mathrm{H}$ oxidase/Racl pathway, producing superoxide in the brain. ${ }^{12,16}$ Chronic oral administration of olmesartan nearly abolishes the pressor response evoked by microinjection of Ang II into the RVLM of SHR. ${ }^{40}$ Supporting our findings, chronic oral treatment with another AT1-receptor blocker, candesartan, attenuates the pressor response induced by microinjection of Ang II into the RVLM. ${ }^{41}$ We measured superoxide production in several brain tissues of olmesartan-treated, non-treated SHRSP and WKY, and the findings supported our measurements by in vivo ESR spectroscopy.

Activation of the brain angiotensin system contributes to the neural mechanisms of hypertension. ${ }^{1-3}$ Circulating Ang II acts on the brain through the circumventricular organ, which lacks a bloodbrain barrier. ${ }^{1-3}$ In addition, a renin-angiotensin system also exists 
a

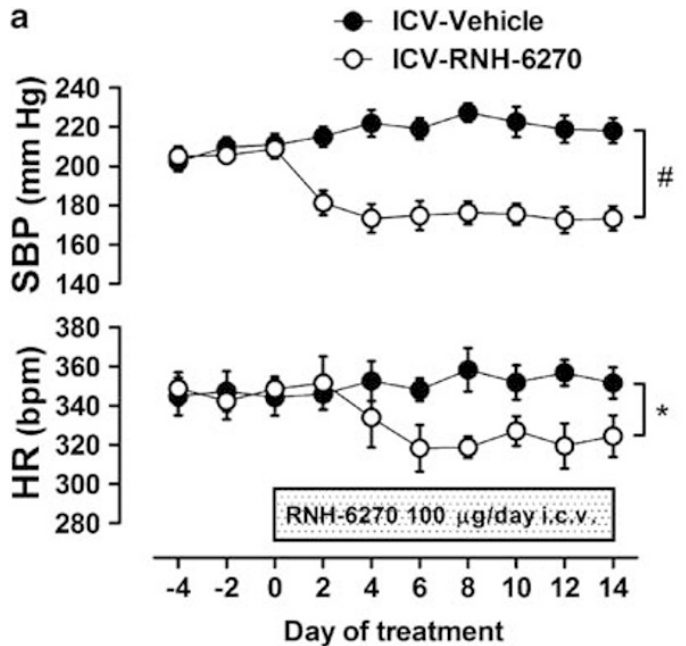

b

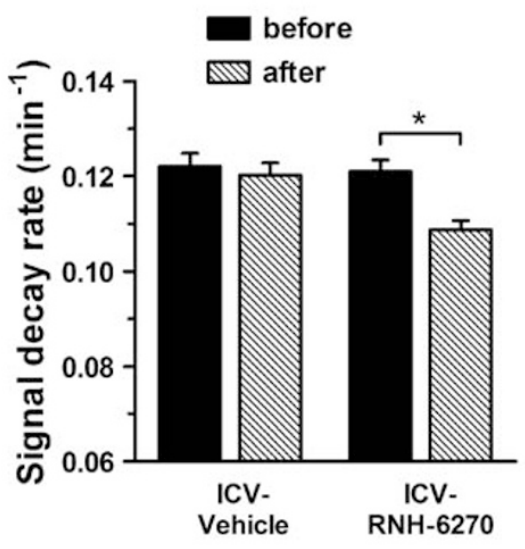

C

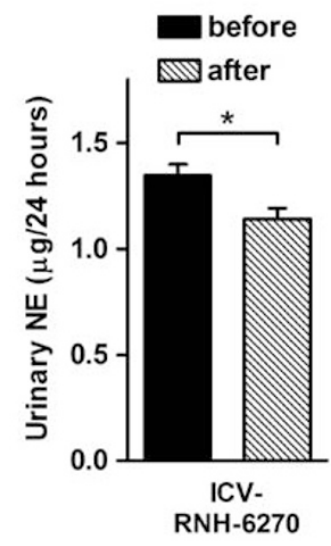

Figure 5 (a) Time course of systolic blood pressure (SBP) and heart rate (HR) in groups that received either intracerebroventricular (ICV) RNH-6270 $\left(100 \mu\right.$ day $\left.^{-1}\right)$ or vehicle. (b) In vivo electron spin resonance signal decay rates before and after treatment in the ICV-vehicle and ICV-RNH-6270 groups. ${ }^{*} P<0.05,{ }^{\#} P<0.001$. (c) Urinary norepinephrine (NE) excretion levels before and after treatment in the ICV-RNH-6270 group.

inside the blood-brain barrier. ${ }^{1-3,8}$ All components of the reninangiotensin system are present in the brain, such as renin, angiotensinogen, angiotensin-converting enzyme, Ang II and AT1 and angiotensin type 2 (AT2) receptors. ${ }^{1-3,8}$ Importantly, AT1 receptors are richly distributed in the paraventricular nucleus of the hypothalamus, SON, nucleus tractus solitarus and RVLM, which are involved in autonomic cardiovascular regulation. ${ }^{1-3}$ AT2 receptors are also distributed in other areas of the brain, such as the inferior olive. ${ }^{5}$ Therefore, we consider that AT1 receptors in specific nuclei are an important target for the effects of olmesartan.

Olmesartan is a powerful AT1-receptor blocker. ${ }^{19,20}$ Our findings support the idea that olmesartan acts within the brain directly or indirectly and reduces oxidative stress in a hypertensive model. Consistent with our findings, chronic administration of olmesartan attenuates the exaggerated pressor response to L-glutamate in the RVLM of SHR. ${ }^{40}$ In addition, in that study, it is shown that the pressor response induced by microinjection of Ang II into the RVLM is diminished in SHR treated with olmesartan. ${ }^{40}$ AT1 receptors are highly expressed in the RVLM ${ }^{17}$ and microinjection of Ang II into the RVLM increases arterial blood pressure through activation of the sympathetic nervous system. ${ }^{42}$ Thus, this finding indicates that orally administered olmesartan inhibits the AT1 receptors within the brain. One might argue that the blood-brain barrier prevents systemically administered olmesartan from acting on the brain. Several reports, however, showed that AT1-receptor blockers act within the brain, as well as in the peripheral vasculature..$^{5-10,43,44}$ Different AT1 blockers may thus act centrally or peripherally and block different AT1 receptors. ${ }^{8,44}$ Lipophilicity and pharmacokinetics are important factors for determining blood-brain barrier permeability. ${ }^{8-10}$ Although olmesartan has a lipophilic property, its active form RNH-6270 has a hydrophilic property and a strong AT1 receptor binding capacity. ${ }^{20,22}$ Approximately $1-2 \mathrm{ng} \mathrm{ml}^{-1}$ of olmesartan was detected in the cerebrospinal fluid in both WKY and SHRSP (data not shown). It is not clear whether this small amount of concentration of olmesartan acted directly on the brain in vivo. Other mechanisms, such as active transport of the drugs through the blood-brain barrier, should be considered, ${ }^{9}$ however, because the hydrophilic AT1-receptor blocker, candesartan, also acts within the brain. ${ }^{6,9}$ In addition, blood-brain barrier disruption might occur in SHRSP, ${ }^{45,46}$ thereby affecting the effect of olmesartan on the brain. ICV administration of RNH-6270, an active form of olmesartan, reduced SBP, HR and urinary NE excretion in association with the reduced oxidative stress in the brain of SHRSP as assessed by the in vivo ESR technique, suggesting that olmesartan has a direct sympatho-inhibitory and antioxidant action on the brain. There is a possible mechanism that orally treated olmesartan acted on the AT1 receptors of the circumventricular organ and area postrema that lacks blood-brain barrier, thereby inhibiting AT1 receptors within the brain through neural pathway., ${ }^{5,4}$ This indirect action of olmesartan could also attenuate oxidative stress in the brain.

Oxidative stress in the brain, particularly in hypertensive patients, is considered to be an important therapeutic target, as well as in other organs. ${ }^{47}$ For example, an AT1-receptor blocker, candesartan, improves cognitive function in hypertensive patients. ${ }^{48}$ In addition, olmesartan improves cognitive function in mice fed a high-salt and cholesterol diet. ${ }^{49}$ Furthermore, brain oxidative stress contributes to the neural mechanisms of hypertension. Antihypertensive drugs or statins reduce oxidative stress in the brain. ${ }^{50-52}$ Although evaluating oxidative stress in the brain in vivo is difficult, particularly in the specific nuclei, this in vivo ESR technique with high-resolution imaging ${ }^{53}$ is promising for future studies.

In conclusion, our results suggest that chronic oral treatment with olmesartan, a powerful and relatively new type of AT1-receptor blocker, has an anti-oxidative effect on the brain in SHRSP assessed by the in vivo ESR method and does not cause reflex-mediated sympathetic activation despite strong blood pressure reduction. Future experiments evaluating the oxidative stress in specific nuclei will be required for determining the role of autonomic nuclei in the reduction of oxidative stress treated with olmesartan.

\section{CONFLICT OF INTEREST}

The authors declare no conflict of interest.

\section{ACKNOWLEDGEMENTS}

This study was supported by a Grant-in-Aid for Scientific Research from the Japan Society for the Promotion of Science (B193290231), a Grant from the Salt Science Research Foundation (No. 0738), and in parts, the Bayer Scholarship for Cardiovascular Research from Japan Cardiovascular Research 
Foundation and the Health and Labor Sciences Research Grant for Comprehensive Research in Aging and Health Labor and Welfare of Japan. We are grateful to Daiichi-Sankyo Co, Tokyo, Japan, for supplying olmesartan and RNH-6270.

1 Phillips MI, Sumners C. Angiotensin II in the central nervous system physiology. Regu Pept 1998; 78: 1-11.

2 McKinley MJ, Albiston AL, Allen AM, Mathai ML, May CN, McAllen RM, Oldfield BJ, Mendelsohn FAO. The brain renin-angiotensin system: location and physiological roles. Int J Biochem Cell Biol 2003; 35: 901-918.

3 Dampney RAL. Functional organization of central pathways regulating the cardiovascular system. Physiol Rev 1994; 74: 323-364.

4 Oshima N, Kumagai H, Onimaru H, Kawai A, Pilowsky PM, ligaya K, Takimoto C, Hayashi K, Saruta T, Itoh H. Monosynaptic excitatory connection from the rostral ventrolateral medulla to sympathetic preganglionic neurons revealed by simultaneous recordings. Hypertens Res 2008; 31: 1445-1454.

5 Saavedra JM. Brain angiotensin II: new developments, unanswered questions and therapeutic opportunities. Cell Mol Neurobiol 2005; 25: 485-512.

6 Seltzer A, Bregonzio C, Armando I, Baiardi G, Saavedra JM. Oral administration of an $\mathrm{AT}_{1}$ receptor antagonist prevents the central effects of angiotensin II in spontaneously hypertensive rats. Brain Res 2004; 1028: 9-18.

7 Zhang J, Leenen FHH. Peripheral administration of $\mathrm{AT}_{1}$ receptor blockers and pressor responses to central angiotensin II and sodium. Can J Physiol Pharmacol 2001; 79: 861-867.

8 Wang JM, Tan J, Leenen FHH. Central nervous system blockade by peripheral administration of $\mathrm{AT}_{1}$ receptor blockers. J Cardiovasc Pharmacol 2003; 41: 593-599.

9 Gohlke P, von Kügelgen S, Jügensen T, Kox T, Rascher W, Culman J, Unger T. Effects of orally applied candesartan cilexetil on central responses to angiotensin II in conscious rats. J Hypertens 2002; 20: 909-918.

10 Gohlke P, Weiss S, Jansen A, Wienen W, Strangier J, Rascher W, Culman J, Unger T. $\mathrm{AT}_{1}$ receptor antagonist telmisartan administered peripherally inhibits central responses to angiotensin II in conscious rats. J Pharmacol Exp Ther 2001; 298: 62-70.

11 Hirooka Y. Role of ROS in brainstem in neural mechanisms of hypertension. Auton Neurosci 2008; 142: 20-24.

12 Peterson JR, Shrama RV, Davisson RL. Reactive oxygen species in the neuropathogenesis of hypertension. Curr Hypertens Rep 2006; 8: 232-241.

13 Kishi T, Hirooka Y, Kimura Y, Ito K, Shimokawa H, Takeshita A. Increased ROS in rostral ventrolateral medulla contribute to neural mechanisms of hypertension in stroke-prone spontaneously hypertensive rats. Circulation 2004; 109: 2357-2362.

14 Kimura $Y$, Hirooka $Y$, Sagara $Y$, Ito K, Kishi T, Shimokawa H, Takeshita A, Sunagawa K. Overexpression of inducible nitric oxide synthase in rostral ventrolateral medulla causes hypertension and sympathoexcitation via an increase in oxidative stress. Circ Res 2005; 96: 252-260.

15 Zimmerman MC, Dunlay RP, Lazartigues E, Zhang Y, Sharma RV, Engelhardt JF, Davisson RL. Requirement for Rac1-dependent NADPH oxidase in the cardiovascular and dipsogenic actions of angiotensin II in the brain. Circ Res 2004; 95: 532-539.

16 Nozoe M, Hirooka Y, Koga Y, Sagara Y, Kishi T, Engelhardt JF, Sunagawa K. Inhibition of Rac1-derived ROS in nucleus tractus solitarius decreases blood pressure and heart rate in stroke-prone spontaneously hypertensive rats. Hypertension 2007; 50: 62-68.

$17 \mathrm{Hu}$ L, Zhu D-N, Yu Z, Wang JQ, Sun Z-J, Yao T. Expression of angiotensin II type 1 $\left(\mathrm{AT}_{1}\right)$ receptor in the rostral ventrolateral medulla in rats. $J$ Appl Physiol 2002; 92 : 2153-2161.

18 Koga Y, Hirooka Y, Araki S, Nozoe M, Kishi T, Sunagawa K. High salt intake enhances blood pressure increase during development of hypertension via oxidative stress in rostral ventrolateral medulla of spontaneously hypertensive rats. Hypertens Res 2008; 31: 2075-2083.

19 Scott LJ, McCormack PL. Olmesartan medoxomil: a review of its use in the management of hypertension. Drugs 2008; 68: 1239-1272.

20 Koike H, Sada T, Mizuno M. In vitro and in vivo pharmacology of olmesartan medoxomil, an angiotensin II type $\mathrm{AT}_{1}$ receptor antagonist. J Hypertens 2001; 19(Suppl 1): S3-S14.

21 Yao L, Kobori H, Rahman M, Seth DM, Shokoji T, Fan Y, Zhang GX, Kimura S, Abe Y, Nishiyama A. Olmesartan improves endothelin-induced hypertension and oxidative stress in rats. Hypertens Res 2004; 27: 493-500.

22 Utsumi H, Muto E, Masuda S, Hamada A. In vivo ESR measurement of free radicals in whole mice. Biochem Biophys Res Commun 1990; 172: 1342-1348.

23 Miura Y, Ozawa T. Noninvasive study of radiation-induced oxidative damage using in vivo electron spin resonance. Free Radic Biol Med 2000; 28: 854-859.

24 Sonta T, Inoguchi T, Matsumoto S, Yasukawa K, Inuo M, Tsubouchi H, Sonoda N, Kobayashi K, Utsumi H, Nawata H. In vivo imaging of oxidative stress in the kidney of diabetic mice and its normalization by angiotensin II type 1 receptor blocker. Biochem Biophys Res Commun 2005; 330: 415-422.

25 Miller Jr FJ, Griendling KK. Functional evaluation of nonphagocytic NAD(P)H oxidases. Methods Enzymol 2002; 353: 220-233.

26 Sano H. Matsumoto K, Utsumi H. Synthesis and imaging of blood-brain-barrier permeable nitroxyl-probes for free radical reactions in brain of living mice. Biochem Mol Biol Int 1997; 42: 641-647.
27 Sano H, Naruse M, Matsumoto K, Oi T, Utsumi H. A new nitroxyl-probe with high retention in the brain and its application for brain imaging. Free Radic Biol Med 2000; 28: 959-969.

28 Anzai K, Saito K, Takeshita K, Takahashi S, Miyazaki H, Shoji H, Lee M-C, Masumizu T, Ozawa T. Assessment of ESR-CT imaging by comparison with autoradiography for the distribution of a blood-brain-barrier permeable spin probe, MC-PROXYL, to rodent brain. Magn Reson Imaging 2003; 21: 765-772.

29 Swartz HM, Sentjurc M, Morse II PD. Cellular metabolism of water-soluble nitroxides: effect on rate of reduction of cell/nitroxide ratio, oxygen concentrations and permeability of nitroxides. Biochim Biophys Acta 1986; 888: 82-91.

30 Takeshita K, Saito K, Ueda J, Anzai K, Ozawa T. Kinetic study on ESR signal decay of nitroxyl radicals, potent redox probes for in vivo ESR spectroscopy, caused by ROS. Biochim Biophys Acta 2002; 1573: 156-164.

31 Kinugawa S, Post H, Kaminski PM, Zhang X, Xu X, Huang H, Recchia A, Ochoa M, Wolin MS, Kaley G, Hintze TH. Coronary microvascular endothelial stunning after acute pressure overload in the conscious dog is caused by oxidant processes: the role of angiotensin II type 1 receptor and NAD(P)H oxidase. Circulation 2003; 108: 2934-2940.

32 Martz D, Rayos G, Schielke GP, Betz AL. Allopurinol and dimethylthiourea reduce brain infarction following middle cerebral artery occlusion in rats. Stroke 1989; 20: 488-494.

33 Giray B, Gürbay A, Hincal F. Cypermethrin-induced oxidative stress in rat brain and liver is prevented by Vitamin E or allopurinol. Toxicol Lett 2001; 118: 139-146.

34 Paxinos G, Watson C. The Rat Brain in Stereotaxic Coordinates. Academic Press: New York, 1998.

35 Ide T, Tsutsui H, Kinugawa S, Suematsu N, Hayashidani S, Ichikawa K, Utsumi H, Machida Y, Egashira K, Takeshita A. Direct evidence for increased hydroxyl radicals originating from superoxide in the failing myocardium. Circ Res 2000; 86: 152-157.

36 Shiba T, Yamato M, Kudou W, Ichikawa K, Yamada K-I, Watanabe T, Utsumi H. Analysis of nitroxyl spin probes in mouse brain by X-band ESR with microdialysis technique. J Pharm Sci 2008; 97: 4101-4107.

37 Lee M-C, Shoji H, Miyazaki H, Yoshino FT, Hori N, Toyoda M, Ikeda Y, Anzai K, Ikota N, Ozawa T. Assessment of oxidative stress in the spontaneously hypertensive rat brain using electron spin resonance (ESR) imaging and in vivo L-band ESR. Hypertens Res 2004; 27: 485-492.

38 Igase M, Kohara K, Nagai T, Miki T, Ferrario CM. Increased expression of angiotensin converting enzyme 2 in conjunction with reduction of neointima by angiotensin II type 1 receptor blockade. Hypertens Res 2008; 31: 553-559.

39 Matsumura K, Averill DB, Ferrario CM. Angiotensin II acts at AT1 receptors in the nucleus of the solitary tract to attenuate the baroreceptor reflex. Am J Physiol 1998; 275: R1611-R1619.

40 Lin Y, Matsumura K, Kagiyama S, Fukuhara M, Fujii K, lida M. Chronic administration of olmesartan attenuates the exaggerated pressor response to glutamate in the rostral ventrolateral medulla of SHR. Brain Res 2005; 1058: 161-166.

41 Tsuchihashi T, Kagiyama S, Matsumura K, Abe I, Fujishima M. Effects of chronic oral treatment with imidapril and TCV-116 on the responsiveness to angiotensin II in ventrolateral medulla of SHR. J Hypertens 1999; 17: 917-922.

42 Hirooka Y, Potts PD, Dampney RAL. Role of angiotensin II receptor subtypes in mediating the sympathoexcitatory effects of exogenous and endogenous angiotensin peptides in the rostral ventrolateral medulla of the rabbit. Brain Res 1997; 772: 107-114.

43 Ye S, Zhong H, Duong VN, Campese V. Losartan reduces central and peripheral sympathetic nerve activity in a rat model of neurogenic hypertension. Hypertension 2002; 39: 1101-1106.

44 Culman J, Blume A, Gohlke P, Unger T. The renin-angiotensin system in the brain: possible therapeutic implications for $\mathrm{AT}_{1}$-receptor blockers. J Hum Hypertens 2002; 16: S64-S70.

45 Ueno M, Sakamoto H, Liao Y-J, Onodera M, Huang C-L, Miyanaka H, Nakagawa T. Blood-brain barrier disruption in the hypothalamus of young adult spontaneously hypertensive rats. Histochem Cell Biol 2004; 122: 131-137.

46 Lippoldt A, Kniesel U, Liebner S, Kalbacher H, Kirsch T, Wolburg H, Haller $\mathrm{H}$. Structural alterations of tight junctions are associated with loss of polarity in strokeprone spontaneously hypertensive rat blood-brain barrier endothelial cells. Brain Res 2000; 885: 251-261.

47 Iwanami J, Mogi M, Iwai M, Horiuchi M. Inhibition of the renin-angiotensin system and target organ protection. Hypertens Res 2009; 32: 229-237.

48 Saxby BK, Harrington F, Wenes KA, McKeith IG, Ford GA. Candesartan and cognitive decline in older patients with hypertension. Neurology 2008; 70: 1858-1866.

49 Mogi M, Tsukuda K, Li J-M, Iwanami J, Min L-J, Sakata A, Fujita T, Iwai M, Horiuchi M. Inhibition of cognitive decline in mice fed a high-salt and cholesterol diet by the angiotensin receptor blocker, olmesartan. Neuropharmacology 2007; 53: 899-905.

50 Hirooka Y, Kimura Y, Nozoe M, Sagara Y, Ito K, Sunagawa K. Amlodipine-induced reduction of oxidative stress in the brain is associated with sympatho-inhibitory effects in stroke-prone spontaneously hypertensive rats. Hypertens Res 2006; 29: 49-56.

51 Kishi T, Hirooka Y, Shimokawa H, Takeshita A, Sunagawa K. Atorvastatin reduces oxidative stress in the rostral ventrolateral medulla of stroke-prone spontaneously hypertensive rats. Clin Exp Hypertens 2008; 30: 1-9.

52 Konno S, Hirooka Y, Araki S, Koga Y, Kishi T, Sunagawa K. Azelnidipine decreases sympathetic nerve activity via antioxidant effect in the rostral ventrolateral medulla of stroke-prone spontaneously hypertensive rats. J Cardiovasc Pharmacol 2008; 52: $555-560$.

53 Utsumi H, Yamada K-I, Ichikawa K, Sakai K, Kinoshita Y, Matsumoto S, Nagai M. Simultaneous molecular imaging of redox reactions monitored by Overhauser-enhanced MRI with ${ }^{14} \mathrm{~N}$ - and ${ }^{15} \mathrm{~N}$-labeled nitroxyl radicals. Proc Natl Acad Sci USA 2006; 103 : $1463-1468$. 